\title{
Metabolically Healthy, but Obese Individuals and Associations with Echocardiographic Parameters and Inflammatory Biomarkers: Results from the CARLA Study
}

This article was published in the following Dove Press journal:

Diabetes, Metabolic Syndrome and Obesity: Targets and Therapy

\author{
Ljupcho Efremov (D) \\ Maria Elena Lacruz ${ }^{2}$ \\ Daniel Tiller ${ }^{3}$ \\ Daniel Medenwald (iD) \\ Karin Halina Greiser ${ }^{4}$ \\ Alexander Kluttig' \\ Andreas Wienke (D) \\ Sebastian Nuding ${ }^{5}$ \\ Rafael Mikolajczyk (iD) \\ 'Institute of Medical Epidemiology, \\ Biostatistics, and Informatics (IMEBI), \\ Interdisciplinary Center for Health \\ Sciences, Medical School of the Martin- \\ Luther-University Halle-Wittenberg, \\ Halle (Saale), Germany; ${ }^{2}$ Clinical Cancer \\ Register, Saxony-Anhalt, Halle (Saale), \\ Germany; ${ }^{3}$ IT Department, Data \\ Integration Center, University Hospital \\ Martin-Luther-University Halle- \\ Wittenberg, Halle (Saale), Germany; \\ ${ }^{4}$ Division of Cancer Epidemiology, \\ German Cancer Research Centre, \\ Heidelberg, Germany; ${ }^{5}$ Department of \\ Internal Medicine III, University Hospital \\ Martin-Luther-University Halle- \\ Wittenberg, Halle (Saale), Germany
}

Correspondence: Rafael Mikolajczyk Institute of Medical Epidemiology, Biostatistics, and Informatics (IMEBI), Interdisciplinary Center for Health Sciences, Medical School of the MartinLuther-University Halle-Wittenberg, Magdeburger Str. 8, Halle (Saale) 06II2, Germany

Email rafael.mikolajczyk@uk-halle.de
Introduction: The research on heterogeneity among obese individuals has identified the metabolically healthy, but obese (MHO) phenotype as a distinct group that does not experience the typical cardiovascular-related diseases (CVD). It is unclear if this group differs with regard to preconditions for CVDs. Our aim was to assess differences in echocardiographic parameters and inflammatory biomarkers between MHO and metabolically healthy, normal weight individuals (MHNW).

Methods: The analyses used data from 1412 elderly participants from a German populationbased cohort study (CARLA), which collected detailed information on demographic, biochemical, and echocardiographic variables. Participants were subdivided into four groups (MHNW, MHO, MUNW (metabolically unhealthy, normal weight) and MUO (metabolically unhealthy, obese)) based on BMI $\geq 30 \mathrm{~kg} / \mathrm{m}^{2}$ (obese or normal weight) and presence of components of the metabolic syndrome. The clinical characteristics of the 4 groups were compared with ANOVA or Chi-Square test, in addition to two linear regression models for 16 echocardiographic parameters. The difference in inflammatory biomarkers (hsCRP, IL-6 and sTNF-RI) between the groups was examined with a multinomial logistic regression model.

Results: The MHO individuals were on average $64.2 \pm 8.4$ years old, with a higher proportion of women (71.6\%), low percentage of smokers, larger waist circumference $(109.3 \pm 10.5 \mathrm{~cm}$ vs $89.1 \pm 10.8 \mathrm{~cm}, \mathrm{p}<0.0001)$ and higher odds ratios for hsCRP, IL- 6 and sTNF-RI compared to MHNW individuals. Linear regression models revealed greater left atrial (LA) diameter (2.73 (95\% CI: 1.35-4.11) mm), LA volume (7.86 (95\% CI: 2.88-12.83) mL), and left ventricular mass index (LVMI) $\left(11.82(95 \% \mathrm{CI}: 4.43-19.22) \mathrm{g} / \mathrm{m}^{1.7}\right)$ in the MHO group compared to the MHNW group.

Conclusion: The MHO phenotype is associated with echocardiographic markers of cardiac remodeling (LA diameter, volume and LVMI) and higher odds ratios for inflammatory biomarkers

Keywords: obesity phenotypes, visceral adipose tissue, cardiac remodeling, inflammatory biomarkers

\section{Introduction}

Population-based studies have identified an obesity subgroup which is hypothesized to have lower risk of cardiovascular system-related complications due to absence of accompanying metabolic disorders - described as having "uncomplicated obesity". ${ }^{1-5}$ These individuals have been designated as obese, but metabolically healthy (MHO)., 
A limited number of studies evaluated the cardiac characteristics of MHO individuals and have reported contradicting findings. Two studies found subtle impairments of left ventricular (LV) systolic ${ }^{8}$ and/or diastolic function, ${ }^{9}$ while one study reported normal myocardial performance. ${ }^{10}$ Mechanisms affecting cardiovascular health in this subset of individuals are still being examined, such as the effect from increased visceral adipose tissue, which is clinically quantified by measuring the waist circumference. ${ }^{11}$ Research on fat storage in different body compartments, specifically the subcutaneous and visceral depots, ${ }^{12}$ and their association with systemic inflammation ${ }^{13}$ requires another evaluation in MHO individuals. Inflammation plays an important role in the onset of cardiovascular disease, with the visceral adipose tissue incriminated as a responsible site for chronic lowgrade inflammation termed "meta-inflammation". ${ }^{4}$ The interplay between inflammation and obesity in the MHO phenotype has only been partially described, usually by only examining the concentration of $\mathrm{C}$-reactive protein (CRP). ${ }^{15}$

Previously published studies have not considered the unique characteristics of obesity in an elderly population, where due to changes in body composition, lifestyle behaviors, physical and immunological parameters, the possibility of preserving metabolic health could have important public health relevance. ${ }^{16}$ Therefore, the aim of the current study was to investigate the myocardial structure and function, as well as, inflammatory biomarkers concentration in 2 specific obesity phenotypes (MHO and metabolically unhealthy obese - MUO), one normal weight, but metabolically unhealthy phenotype (MUNW) and compare them to the metabolically healthy and normal weight (MHNW) participants enrolled in an elderly, populationbased cohort. We hypothesized that MHO individuals will show subclinical perturbations in their cardiac structure and increased levels of inflammatory biomarkers, despite the absence of the metabolic syndrome.

\section{Methods}

\section{Study Population}

Data from the first follow-up examination of the CArdiovascular Risk Factors, Living and Ageing in Halle (CARLA), were used for analysis. Details on the study design and methods have been described elsewhere. ${ }^{17,18}$ In brief, CARLA is a cohort study of elderly inhabitants of the city of Halle (Saale) in Germany with a focus on examining risk factors for cardiovascular diseases. The baseline examination was conducted between December 2002 and January 2006 and included 1779 participants (812 women and 967 men, between 45 and 83 years old). The first followup (CARLA-1) was conducted from March 2007 to March 2010, in which 1436 (45\% women) individuals participated. During CARLA-1 the enrolled participants underwent deep cardiovascular ultrasound phenotyping.

The study was approved by the Ethics Committee of the Medical Faculty of the Martin-Luther University HalleWittenberg and by the State Data Privacy Commissioner of Saxony-Anhalt and conforms with the Declaration of Helsinki. All participants gave their written informed consent. After removing individuals with missing information $(n=24)$ on body mass index (BMI), blood pressure (BP), biochemical parameters or medication history, the final analytical sample comprised 1412 participants.

\section{Clinical and Biochemical Data Collection}

Data were collected with standardized computer-assisted personal interviews and medical examinations by trained study nurses. Information on demographic characteristics, medical history, and lifestyle factors were recorded, including questions on current smoking and sport activity. In addition, each participant's BP, height, weight and heart rate were measured. BP was calculated as the mean of the second and third measurement out of three consecutive measurements. BMI was calculated as weight in kilograms divided by height in meters squared. Waist circumference was measured at the midpoint between the anterior superior iliac spine and the lower edge of the ribcage. The estimated glomerular filtration rate (eGFR), calculated with the Chronic Kidney Disease Epidemiology Collaboration formula, was used to assess renal function. ${ }^{19}$

Venous blood samples were collected, and glucose, total cholesterol, high-density lipoprotein (HDL) cholesterol, triglycerides and creatinine levels were measured using automated enzymatic methods in a single certified laboratory. While participants were not required to fast before blood collection, the time from last meal or beverage intake was more than 2 hours in $95 \%$ of participants. Serum high-sensitivity CRP (hsCRP) was measured using a high-sensitivity immunoturbidimetric method (CRP [Latex] HS, Roche, Mannheim, Germany) on a Hitachi auto-analyzer (Roche Diagnostics, Mannheim, Germany). Measurement of soluble tumor necrotic factor receptor I (sTNF-RI) concentration was carried out using the Human sTNF-RI/TNFSF1A Immunoassay Quantikine ELISA kit (BioTek, Bad Friedrichshall, Germany), while 
interleukin-6 (IL-6) measurements were carried out using the IL-6 electrochemiluminescence immunoassay (ECLIA) kit (Roche Diagnostics, Rotkreuz, Switzerland).

\section{Echocardiographic Assessment}

Doppler echocardiographic examination was performed by a trained and certified study nurse, and the stored echocardiographic recordings were subsequently evaluated by a trained physician. All echocardiographic examinations, including standard M-mode, two-dimensional and TDI echocardiography, were performed using the GE Vivid ultrasound system (GE Vivid, GE Vingmed, Norway) according to the guidelines from the American Society of Echocardiography (ASE). ${ }^{20}$ Left ventricular ejection fraction (LV EF) was estimated by the modified Simpson's rule. $^{21}$ The ASE-cube formula indexed to height raised by an exponent of 1.7 was used to calculate the LV mass index (LVMI) as recommended by a previous publication. ${ }^{22}$ Trans-mitral Doppler parameters included mitral early (E) and late (A) wave velocities, from which the E/A ratio was calculated. Additionally, tissue Doppler measurements were obtained including mitral annular early diastolic (e') and late (a') diastolic velocities from which the E/E' ratio, an index of left ventricular filling pressure, was calculated.

\section{Definition of the Phenotypes}

We considered four components of the metabolic syndrome $^{23} 1$ ) systolic $\mathrm{BP} \geq 130 \mathrm{mmHg}$ or diastolic BP $\geq 85 \mathrm{mmHg}$, or intake of antihypertensive medication; 2) plasma glucose $\geq 5.6 \mathrm{mmol} / \mathrm{L}$ or intake of antidiabetic medication; 3) HDL cholesterol $<1.0 \mathrm{mmol} / \mathrm{L}$ for men and $<1.3 \mathrm{mmol} / \mathrm{L}$ for women, or dyslipidemia treatment, and 4) triglycerides $\geq 1.7 \mathrm{mmol} / \mathrm{L}$. Participants with two or more components were defined as metabolically unhealthy; the remaining as metabolically healthy. Individuals with $\mathrm{BMI} \geq 30 \mathrm{~kg} / \mathrm{m}^{2}$ were considered obese, according to the World Health Organization classification. Status of metabolic health and obesity categories were combined to create the four phenotypes: MHNW, MHO, MUNW, and MUO.

\section{Statistical Methods}

Data are presented as mean $\pm \mathrm{SD}$ or median (first quartile (Q1) - third quartile (Q3)) for continuous variables and absolute and relative frequencies for categorical variables. For metric variables, comparisons among the 4 groups were performed with a one-way analysis of variance (ANOVA). In case of inequality of group variances, as ascertained by the Levene's test, Welch ANOVA p-values are reported. For the variables with non-normally distributed residuals, the Kruskal-Wallis test was used. Post-hoc tests, such as the Games-Howell test or the Dunn's test, with the MHNW phenotype as the reference group, were used to determine between-group differences. For categorical variables, the Chi-square or Fisher's exact test was used.

To assess the association between the 4 phenotypes and the echocardiographic parameters, two general linear regression models were applied for each echocardiographic variable. The echocardiographic variables followed a normal distribution and the linear regression model was deemed the most fitting to analyze the parameters in their original units. The first model adjusted for age and sex, while the second model additionally adjusted for current smoking, sport activity, and medication use (antihypertensive, lipid-lowering and anti-diabetic medication). Directed acyclic graphs (DAGs) were used to identify the minimally sufficient adjustment set for the second model by using the program DAGitty (Johannes Textor, Theoretical Biology \& Bioinformatics Group, University of Utrecht, The Netherlands). The significance threshold was $\alpha=0.05$, however, to account for multiple testing the significance level was corrected by the Bonferroni method, setting the threshold at $\alpha=0.003$ (given 16 variables). Model fit was assessed using quantile-quantile (QQ) plots and standardized residuals against predicted means plot.

The associations between inflammatory biomarkers and the 4 phenotypes were analyzed with a multinomial logistic regression model to examine the difference in risk of inflammation for the groups in reference to the MHNW group. We used the logistic model to calculate odds ratios with the biomarkers as exposure, in line with other studies studying inflammation. The model was only adjusted for age and sex, in order to avoid overfitting, taking into account the small sample size. The biomarker measurements were logarithmically transformed to the base of 2 due to skewed distributions. Statistical analyses were performed with SAS 9.4 (SAS Institute, Cary, NC, USA).

\section{Missing Data}

After examining the missing data pattern of the echocardiographic variables, no regular pattern was observed; hence, the missing data were assumed to be missing at random (MAR). The percentage of missing values ranged from $3 \%$ for LA diameter and $6 \%$ for LA volume to $19 \%$ for LV posterior wall diameter and $30 \%$ for 
interventricular septal diameter. The missing values were imputed using the Markov Chain Monte Carlo (MCMC) imputation method which assumes that all variables in the imputation model have a joint multivariate normal distribution with defined minimum and maximum values for each variable as present in the sample population. ${ }^{24,25}$ Using the PROC MI procedure and the NIMPUTE=PCTMISSING option, 44 imputed datasets were created. Participants' clinical and biochemical characteristics were used as auxiliary variables to improve the quality of imputed values generated with the imputation model.

\section{Results}

\section{Clinical Characteristics of Participants}

In total, one third of the CARLA-1 participants were obese $(30.38 \%)$. Sixty participants (4\%) were classified in the MHO group, 345 (24\%) in the MHNW group, 638 (45\%) in the MUNW group, and $369(26 \%)$ in the MUO group (Table 1). The MHO individuals had a mean age of $64.2 \pm 8.4$ years with a high proportion of women (71.6\%). When this group was compared to the reference (MHNW) phenotype, differences were found in larger waist circumference $(p<0.0001)$ and higher concentrations of inflammatory biomarkers (hsCRP, $\mathrm{p}<0.0001$; IL-6, $\mathrm{p}<0.0001$; and sTNF-R1,

Table I Demographic and Clinical Characteristics of Study Participants

\begin{tabular}{|c|c|c|c|c|c|}
\hline Characteristics & MHNW (n=345) & MHO $(n=60)$ & MUNW (n=638) & MUO (n=369) & p value \\
\hline \multicolumn{6}{|c|}{ Continuous variables Mean $\pm \mathrm{SD} /$ median (IQR) } \\
\hline Age, years & $64.0 \pm 9.6$ & $64.2 \pm 8.4$ & $68.9 \pm 9.7^{*}$ & $67.7 \pm 9.0^{*}$ & $<0.0001$ \\
\hline BMI, $\mathrm{kg} / \mathrm{m}^{2}$ & $25.0 \pm 2.7$ & $34.1 \pm 3.4^{*}$ & $26.4 \pm 2.4^{*}$ & $33.8 \pm 3.8 *$ & $<0.0001$ \\
\hline Waist circumference, $\mathrm{cm}$ & $89.1 \pm 10.9$ & $109.3 \pm 10.5^{*}$ & $96.4 \pm 9.6^{*}$ & $112.9 \pm 10.4^{*}$ & $<0.0001$ \\
\hline Systolic BP, mmHg & $130.9 \pm 17.9$ & $133.7 \pm 17.4$ & $139.3 \pm 19.9 *$ & $139.9 \pm 19.6 *$ & $<0.0001$ \\
\hline Diastolic BP, mmHg & $79.5 \pm 9.1$ & $80.9 \pm 9.0$ & $80.1 \pm 10.8$ & $79.9 \pm 10.3$ & 0.68 \\
\hline Heart rate, beats/min & $69.2 \pm 10.2$ & $70.5 \pm 10.5$ & $68.4 \pm 11.0$ & $68.9 \pm 11.5$ & 0.41 \\
\hline Cholesterol, $\mathrm{mmol} / \mathrm{L}$ & $5.6 \pm 0.9$ & $5.6 \pm 1.0$ & $5.4 \pm 1.1 *$ & $5.3 \pm 1.0 *$ & 0.0002 \\
\hline $\mathrm{HDL}, \mathrm{mmol} / \mathrm{L}$ & $1.6 \pm 0.4$ & $1.5 \pm 0.3$ & $1.3 \pm 0.4^{*}$ & $1.2 \pm 0.3^{*}$ & $<0.0001$ \\
\hline Triglycerides, $\mathrm{mmol} / \mathrm{L}$ & I.2(0.9-I.5) & I.3 (I.I-I.5) & $1.9(1.4-2.6)^{*}$ & $1.9(1.4-2.7)^{*}$ & $<0.0001$ \\
\hline Apo AI, g/L & $1.7 \pm 0.3$ & $1.6 \pm 0.2$ & $1.5 \pm 0.3^{*}$ & $1.4 \pm 0.2^{*}$ & $<0.0001$ \\
\hline Apo B, g/L & $0.89 \pm 0.2$ & $0.88 \pm 0.2$ & $0.93 \pm 0.2$ & $0.95 \pm 0.2 *$ & 0.007 \\
\hline Glucose, $\mathrm{mmol} / \mathrm{L}$ & $5.1(4.8-5.4)$ & $5.2(5.0-5.4)$ & $5.6(5.1-6.1)^{*}$ & $5.8(5.2-6.8)^{*}$ & $<0.0001$ \\
\hline $\mathrm{HbA}_{\mathrm{Ic}}, \%$ & $5.5 \pm 0.4$ & $5.7 \pm 0.4$ & $5.9 \pm 0.8^{*}$ & $6.2 \pm 0.9^{*}$ & $<0.000$ I \\
\hline hsCRP, mg/L & I.I (0.7-2.I) & $3.2(1.7-5.1)^{*}$ & I.5 (0.9-3.0)* & $2.8(1.6-5.4)^{*}$ & $<0.0001$ \\
\hline IL-6, pg/mL & $1.8(1.0-2.9)$ & $3.1(2.4-4.7)^{*}$ & $2.3(1.0-3.8)^{*}$ & $3.2(2.3-5.1)^{*}$ & $<0.0001$ \\
\hline \multirow[t]{2}{*}{ sTNF-RI, pg/mL } & 1078.1 & $1281.8^{*}$ & $1201.5^{*}$ & 1286.9* & $<0.0001$ \\
\hline & $(946.9-1266.1)$ & $(1140.1-1452.3)$ & $(1052.6-1437.2)$ & $(1135.3-1574.7)$ & \\
\hline NT-proBNP, pg/mL & $86.5(52.2-161.2)$ & $85.3(41.6-157.1)$ & $125.9 *(62.1-244.3)$ & $119.2 *(59.1-236.6)$ & $<0.0001$ \\
\hline$\gamma$-GT, ukat/L & $0.4(0.3-0.5)$ & $0.4(0.3-0.6)$ & $0.4(0.3-0.7)^{*}$ & $0.5(0.4-0.8)^{*}$ & $<0.0001$ \\
\hline eGFR, $\mathrm{mL} / \mathrm{min} / 1.73 \mathrm{~m}^{2}$ & $85.1 \pm 15.7$ & $81.8 \pm 16.6$ & $78.6 \pm 18.3^{*}$ & $78.1 \pm 18.3^{*}$ & $<0.0001$ \\
\hline \multicolumn{6}{|c|}{ Categorical variables n (\%) } \\
\hline Women & $186(53.9)$ & $43(71.6)$ & $235(36.8)$ & $167(45.3)$ & $<0.0001$ \\
\hline Current sport activity & $186(53.9)$ & $34(56.7)$ & $293(45.9)$ & I 47 (39.8) & 0.0008 \\
\hline Current smokers & $68(19.7)$ & 7 (II.7) & $101(15.8)$ & $45(I 2.2)$ & 0.03 \\
\hline University education & $111(32.2)$ & $10(16.7)$ & I8I (28.4) & $90(24.4)$ & 0.02 \\
\hline Diabetes medication & $3(0.9)$ & $\mathrm{I}(\mathrm{I} .7)$ & $97(15.2)$ & $96(26.0)$ & $<0.0001$ \\
\hline $\begin{array}{l}\text { Antihypertensive } \\
\text { medication }\end{array}$ & $108(3 \mid .3)$ & $29(48.3)$ & $463(72.6)$ & $307(83.2)$ & $<0.0001$ \\
\hline Lipid-lowering medication & $7(2.0)$ & $2(3.3)$ & $217(34.0)$ & $116(31.4)$ & $<0.0001$ \\
\hline
\end{tabular}

Notes: Results presented as mean \pm SD for normally distributed residuals with one-way ANOVA p-values reported, when there was equal variance between groups, or Welch ANOVA. p-values reported, when variance was unequal. Frequencies with percentages for categorical variables (Chi-square or Fisher's exact test). Non-normally distributed residuals reported with median and IQR ( $\mathrm{p}$-value from Kruskal-Wallis test). Games-Howell or Tukey-Kramer post-hoc test (after ANOVA) and Dunn's post-hoc test (after Kruskal-Wallis). * significant difference $(p<0.05)$ when compared to the reference group (MHNW).

Abbreviations: MHNW, metabolically healthy, normal weight; MHO, metabolically healthy obese; MUNW, metabolically unhealthy, normal weight; MUO, metabolically unhealthy obese; IQR, interquartile range; SD, standard deviation. 
$\mathrm{p}<0.0001)$. Interestingly, the MHO group had the highest reported proportion for current sport activity $(56.7 \%)$ and lowest proportion of current smokers (11.7\%). The MUNW group had an average age of $68.9 \pm 9.7$ years and was characterized with larger waist circumference $(\mathrm{p}<0.0001)$, higher concentration of NT-proBNP $(\mathrm{p}<0.0001)$, and higher concentration of inflammatory biomarkers (hsCRP, $\mathrm{p}<0.001$; IL-6, $<<0.0001$; and sTNF-R1, $\mathrm{p}<0.0001$ ), but lower eGFR $(\mathrm{p}<0.0001)$ when compared to the reference phenotype. As expected, the MUO group, with average age $67.7 \pm 9.0$ years, had the worst profile with presence of many traditional cardiovascular risk factors. The MUO group had the highest proportion of anti-diabetic (26\%) and antihypertensive medication (83.2\%) use, with the exception of lipid-lowering therapy, which was higher in the MUNW group (34\%).

\section{Associations with Echocardiographic Parameters}

Considering cardiac morphology and function, the MHO phenotype was associated with greater left atrial (LA) diameter $(\mathrm{p}=0.003)$ and LA volume $(\mathrm{p}=0.01)$, as well as greater LVMI $(\mathrm{p}=0.01)$ compared to the MHNW group (Table 2, Figure 1). The parameters for diastolic function, E/e' ratio, E/A ratio and mitral e', and the parameter for systolic function (EF), were not different between the MHO and MHNW groups. In the MUNW group differences for LV structural parameters were observed, such as larger septal thickness $(\mathrm{p}<0.0001)$, posterior wall thickness $(p=0.001)$ and higher $E / e^{\prime}(p<0.0001)$, compared to the reference group. Finally, the MUO group was characterized with higher measurements for almost all echocardiographic parameters compared to the MHNW group.

Differences persisted even after age and sex adjustment and adjustment for additional confounders (Table 3, Models 1 and 2). MHO individuals had greater LA diameter $(2.73$ (95\% CI: 1.35 to 4.11$) \mathrm{mm})$, LA volume (7.86 (95\% CI: 2.88 to 12.83$) \mathrm{mL})$ and LVMI (11.82 (95\% CI: 4.43 to 19.22$) \mathrm{g} / \mathrm{m}^{1.7}$ ) compared to MHNW individuals. The MUNW group, reflecting participants with absence of obesity, but presence of metabolic abnormalities, was associated with greater LA diameter (1.39 (95\% CI: 0.65 to 2.13$) \mathrm{mm})$, LVMI (5.90 (95\% CI: 2.04 to 9.76$\left.) \mathrm{g} / \mathrm{m}^{1.7}\right)$, and interventricular septal diameter

Table 2 Echocardiographic Characteristics of Study Participants

\begin{tabular}{|c|c|c|c|c|c|}
\hline Characteristics & MHNW (n=345) & MHO $(n=60)$ & MUNW (n=638) & MUO (n=369) & $\mathrm{p}$ value \\
\hline \multicolumn{6}{|l|}{ LA structure } \\
\hline LA diameter, mm & $37.4 I \pm 5.4$ & $39.98 \pm 5.3^{*}$ & $40.40 \pm 5.3^{*}$ & $43.20 \pm 5.4^{*}$ & $<0.0001$ \\
\hline LA volume, $\mathrm{mL}$ & $35.25 \pm 18.3$ & $43.06 \pm 18.5 *$ & $39.85 \pm 18.9 *$ & $48.32 \pm 18.9 *$ & $<0.0001$ \\
\hline \multicolumn{6}{|l|}{ LV structure } \\
\hline Mass/height ${ }^{1.7}, \mathrm{~g} / \mathrm{m}^{1.7}$ & $74.89 \pm 26.5$ & $85.89 \pm 27.4^{*}$ & $87.15 \pm 27.8^{*}$ & $98.79 \pm 26.5^{*}$ & $<0.0001$ \\
\hline Septal thickness, mm & $10.75 \pm 2.2$ & $11.30 \pm 2.4$ & $11.58 \pm 2.4^{*}$ & $11.84 \pm 2.4^{*}$ & $<0.0001$ \\
\hline Posterior wall thickness, $\mathrm{mm}$ & $10.34 \pm 2.3$ & $10.69 \pm 2.3$ & $10.91 \pm 2.2^{*}$ & $11.29 \pm 2.4^{*}$ & $<0.0001$ \\
\hline Diastolic volume, $\mathrm{mL}$ & $89.2 \pm 37.5$ & $98.1 \pm 38.4$ & $95.1 \pm 37.8$ & $106.2 \pm 38.5^{*}$ & $<0.0001$ \\
\hline Systolic volume, $\mathrm{mL}$ & $34.0 \pm 17.4$ & $36.0 \pm 17.8$ & $36.7 \pm 17.6$ & $40.9 \pm 18.0^{*}$ & $<0.0001$ \\
\hline Diastolic diameter, $\mathrm{mm}$ & $46.85 \pm 6.7$ & $48.52 \pm 7.3$ & $48.89 \pm 7.3^{*}$ & $51.38 \pm 7.0^{*}$ & $<0.0001$ \\
\hline Systolic diameter, $\mathrm{mm}$ & $29.32 \pm 6.5$ & $30.02 \pm 7.2$ & $30.65 \pm 6.6 *$ & $32.40 \pm 6.7^{*}$ & $<0.0001$ \\
\hline \multicolumn{6}{|l|}{ LV systolic function } \\
\hline Ejection fraction, \% & $60.61 \pm 7.0$ & $61.62 \pm 7.2$ & $60.24 \pm 7.0$ & $60.34 \pm 7.3$ & 0.49 \\
\hline \multicolumn{6}{|l|}{ LV diastolic function } \\
\hline E/A ratio & $1.06 \pm 0.4$ & $1.03 \pm 0.4$ & $1.02 \pm 0.4$ & $0.99 \pm 0.4$ & 0.19 \\
\hline E/e' ratio & $10.40 \pm 4.5$ & $11.08 \pm 4.5$ & $11.85 \pm 4.4 *$ & $12.59 \pm 4.6^{*}$ & $<0.0001$ \\
\hline E peak, $\mathrm{cm} / \mathrm{s}$ & $66.2 \pm 18.0$ & $68.42 \pm 17.8$ & $66.0 \pm 17.9$ & $70.37 \pm 18.4^{*}$ & 0.001 \\
\hline A peak, $\mathrm{cm} / \mathrm{s}$ & $66.0 \pm 20.9$ & $68.57 \pm 20.5$ & $70.0 \pm 20.8^{*}$ & $75.6 \pm 21.2^{*}$ & $<0.0001$ \\
\hline e' peak, $\mathrm{cm} / \mathrm{s}$ & $6.78 \pm 1.8$ & $6.52 \pm 1.9$ & $5.97 \pm 1.8^{*}$ & $5.91 \pm 1.8^{*}$ & $<0.0001$ \\
\hline a' peak, cm/s & $9.58 \pm 2.3$ & $9.62 \pm 2.3$ & $9.46 \pm 2.3$ & $9.26 \pm 2.3$ & 0.27 \\
\hline
\end{tabular}

Notes: Results presented as mean \pm SD for normally distributed residuals, difference in means ANOVA for equal variance or Welch ANOVA for unequal variance with Tukey-Kramer post-hoc test. * significant difference $(p<0.05)$ when compared to the reference group $(M H N W)$.

Abbreviations: MHNW, metabolically healthy, normal weight; MHO, metabolically healthy obese; MUNW, metabolically unhealthy, normal weight; MUO, metabolically unhealthy obese; LA, left atrium; LV, left ventricle; SD, standard deviation. 

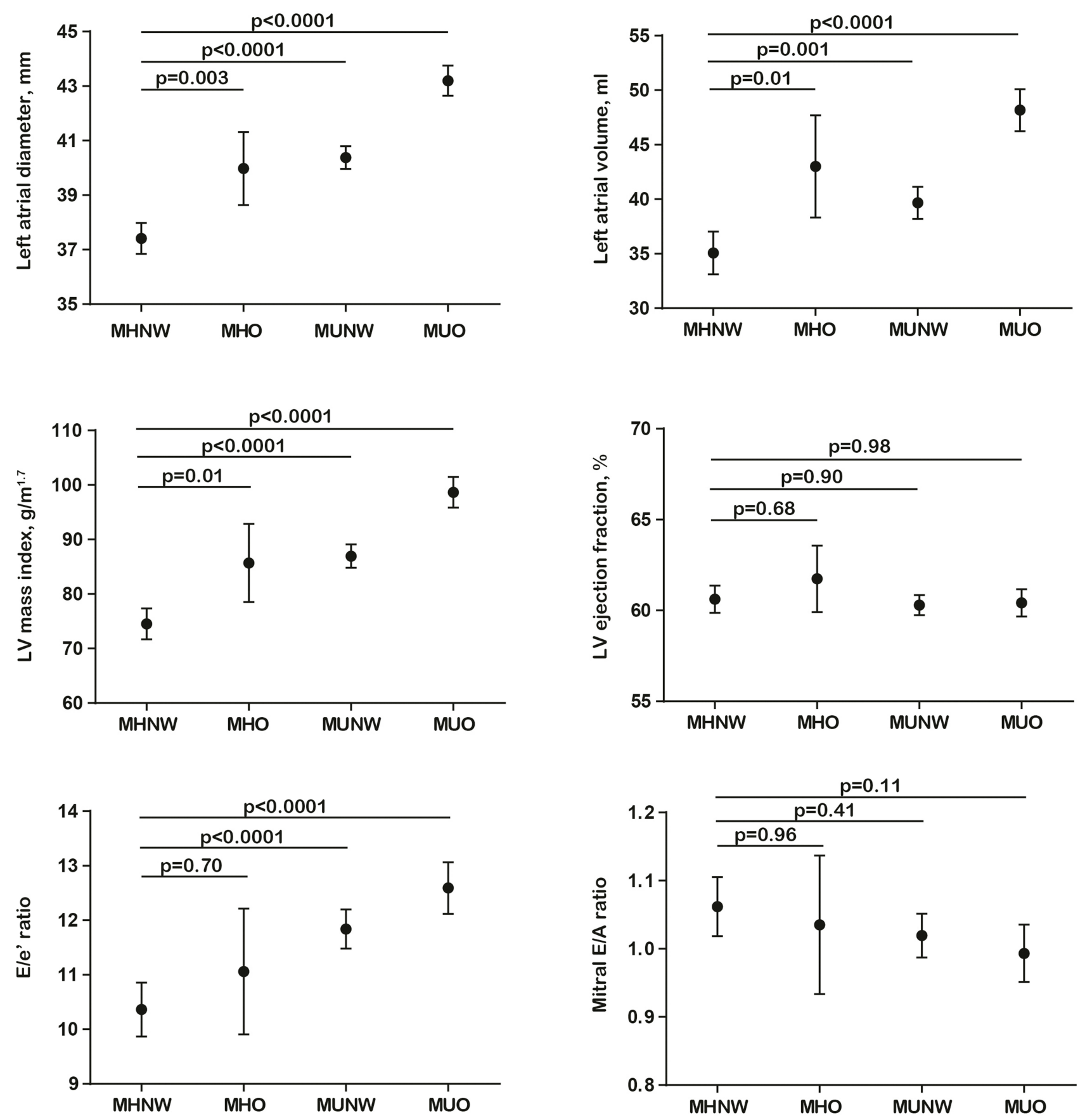

Figure I Differences in means with $95 \% \mathrm{Cl}$ for selected echocardiographic parameters.

Abbreviations: MHNW, metabolically healthy, normal weight; MHO, metabolically healthy obese; MUNW, metabolically unhealthy, normal weight; MUO, metabolically unhealthy obese; LV, left ventricle; $\mathrm{Cl}$, confidence interval.

(0.59 (95\% CI: 0.27 to 0.91$) \mathrm{mm})$, but lower e' peak $(-0.39(95 \%$ CI: -0.64 to -0.14$) \mathrm{cm} / \mathrm{s})$. In the MUO group, where both obesity and metabolic abnormalities were present, an association with LV diastolic dysfunction parameters (greater LA volume, LA diameter, LVMI and E/e' ratio) was more evident.

\section{Associations with Inflammatory Biomarkers}

Odds ratios for each phenotype were calculated, after age and sex adjustment (Figure 2, Model 1), with the MHNW group as reference. The MHO group had higher odds ratios for hsCRP (2.44 (95\% CI: 1.83-3.24)), IL-6 (2.02 (95\% CI: 
Table 3 Multivariable-Adjusted Regression Models for Echocardiographic Parameters

\begin{tabular}{|c|c|c|c|c|c|c|c|}
\hline $\begin{array}{l}\text { Echocardiographic } \\
\text { Parameters }\end{array}$ & $\begin{array}{l}\text { MHNW } \\
(n=345)\end{array}$ & MHO $(n=60)$ & p-value & MUNW (n=638) & p-value & MUO (n=369) & p-value \\
\hline \multicolumn{8}{|l|}{ LA diameter, mm } \\
\hline Model I & Reference & 3.06 (1.67 to 4.46$)$ & $<0.0001$ & 2.08 (1.39 to 2.77$)$ & $<0.0001$ & $5.24(4.48$ to 6.00$)$ & $<0.0001$ \\
\hline Model 2 & Reference & 2.73 (I.35 to 4.11$)$ & $<0.0001$ & $1.39(0.65$ to 2.13$)$ & 0.0002 & 4.31 (3.48 to 5.15$)$ & $<0.0001$ \\
\hline \multicolumn{8}{|l|}{ LA volume, $\mathrm{mL}$} \\
\hline Model I & Reference & 8.78 (3.77 to 13.79) & 0.0006 & $2.59(0.13$ to 5.04$)$ & 0.03 & II.80 (9.08 to 14.52$)$ & $<0.0001$ \\
\hline Model 2 & Reference & 7.86 (2.88 to 12.83$)$ & 0.002 & $0.19(-2.44$ to 2.83$)$ & 0.88 & 8.81 (5.84 to II.79) & $<0.0001$ \\
\hline \multicolumn{8}{|l|}{ LVMI, $g / \mathrm{m}^{1.7}$} \\
\hline Model I & Reference & 12.84 (5.45 to 20.23$)$ & 0.0007 & $8.7 \mid(5.1 \mid$ to 12.30$)$ & $<0.0001$ & $\begin{array}{l}21.70(17.81 \text { to } \\
25.59)\end{array}$ & $<0.000$ I \\
\hline Model 2 & Reference & II.82 (4.43 to 19.22) & 0.001 & $5.90(2.04$ to 9.76$)$ & 0.002 & $\begin{array}{l}\text { I8.2I (13.88 to } \\
22.54)\end{array}$ & $<0.0001$ \\
\hline \multicolumn{8}{|l|}{ IVSd, mm } \\
\hline Model I & Reference & 0.68 (0.03 to 1.33$)$ & 0.03 & 0.62 (0.33 to 0.92$)$ & $<0.0001$ & 0.97 (0.64 to I.29) & $<0.0001$ \\
\hline Model 2 & Reference & $0.64(-0.007$ to 1.28$)$ & 0.05 & 0.59 (0.27 to 0.91$)$ & 0.0003 & 0.89 (0.54 to 1.25$)$ & $<0.0001$ \\
\hline \multicolumn{8}{|l|}{ LVPWd, mm } \\
\hline Model I & Reference & $0.50(-0.12$ to 1.13$)$ & 0.11 & $0.34(0.04$ to 0.65$)$ & 0.02 & $0.82(0.47$ to 1.16$)$ & $<0.0001$ \\
\hline Model 2 & Reference & $0.47(-0.15$ to 1.10$)$ & 0.13 & 0.33 (0.002 to 0.66$)$ & 0.04 & 0.80 (0.43 to 1.18$)$ & $<0.0001$ \\
\hline \multicolumn{8}{|l|}{ LVVd, mL } \\
\hline Model I & Reference & $13.02(2.94$ to 23.10$)$ & 0.01 & $4.63(-0.18$ to 9.45$)$ & 0.05 & I6.94 (II.64 to 22.23$)$ & $<0.0001$ \\
\hline Model 2 & Reference & $12.42(2.30$ to 22.55$)$ & 0.01 & $3.03(-2.21$ to 8.28$)$ & 0.25 & $15.0 \mid$ (9.|I to 20.9I) & $<0.0001$ \\
\hline \multicolumn{8}{|l|}{ LVVs, mL } \\
\hline Model I & Reference & $3.68(-1.06$ to 8.42$)$ & 0.12 & $1.91(-0.36$ to 4.19$)$ & 0.09 & 6.69 (4.17 to 9.22$)$ & $<0.0001$ \\
\hline Model 2 & Reference & $3.52(-1.24$ to 8.28$)$ & 0.14 & $1.05(-1.43$ to 3.54$)$ & 0.40 & 5.75 (2.94 to 8.56$)$ & $<0.0001$ \\
\hline \multicolumn{8}{|l|}{ LVDd, $\mathbf{m m}$} \\
\hline Model I & Reference & $2.44(0.55-4.32)$ & 0.01 & $1.39(0.48$ to 2.30$)$ & 0.002 & 4.21 (3.21 to 5.22$)$ & $<0.0001$ \\
\hline Model 2 & Reference & $2.20(0.33$ to 4.07$)$ & 0.02 & $0.66(-0.30$ to 1.63$)$ & 0.17 & 3.34 (2.23 to 4.45$)$ & $<0.0001$ \\
\hline \multicolumn{8}{|l|}{ LVDs, mm } \\
\hline Model I & Reference & I.31 $(-0.58$ to 3.20$)$ & 0.17 & $0.76(-0.09$ to 1.61$)$ & 0.08 & $2.80(1.83$ to 3.76$)$ & $<0.0001$ \\
\hline Model 2 & Reference & $1.19(-0.68$ to 3.08$)$ & 0.21 & $0.07(-0.84$ to 0.98$)$ & 0.87 & 2.02 (0.97 to 3.07 ) & 0.0002 \\
\hline \multicolumn{8}{|l|}{$E F, \%$} \\
\hline Model I & Reference & $0.90(-1.07$ to 2.88$)$ & 0.37 & $-0.07(-1.01$ to 0.85$)$ & 0.86 & $-0.08(-1.14$ to 0.98$)$ & 0.88 \\
\hline Model 2 & Reference & $0.82(-I .16$ to $2.8 I)$ & 0.41 & $0.04(-0.97$ to 1.06$)$ & 0.93 & $\begin{array}{l}-0.0001(-1.18 \text { to } \\
1.18)\end{array}$ & 0.99 \\
\hline \multicolumn{8}{|l|}{ E/A ratio } \\
\hline Model I & Reference & $-0.01(-0.12$ to 0.09$)$ & 0.77 & $-0.006(-0.05$ to 0.04$)$ & 0.80 & $-0.03(-0.09$ to 0.01$)$ & 0.19 \\
\hline Model 2 & Reference & $-0.02(-0.13$ to 0.08$)$ & 0.63 & $-0.02(-0.08$ to 0.02$)$ & 0.32 & $\begin{array}{l}-0.06(-0.12 \text { to } \\
-0.001)\end{array}$ & 0.05 \\
\hline \multicolumn{8}{|l|}{ E/e' ratio } \\
\hline Model I & Reference & $0.56(-0.63$ to 1.75$)$ & 0.35 & $0.93(0.35$ to $1.5 \mathrm{I})$ & 0.001 & 1.78 (1.14 to 2.42$)$ & $<0.0001$ \\
\hline Model 2 & Reference & $0.39(-0.79$ to 1.58$)$ & 0.51 & $0.43(-0.19$ to 1.05$)$ & 0.17 & $1.03(0.33$ to 1.74$)$ & 0.003 \\
\hline \multicolumn{8}{|l|}{ E peak, $\mathrm{cm} / \mathrm{s}$} \\
\hline Model I & Reference & $1.65(-3.22$ to 6.52$)$ & 0.50 & $0.39(-2.01$ to 2.80$)$ & 0.74 & $4.46(1.77$ to 7.14$)$ & 0.001 \\
\hline Model 2 & Reference & $1.15(-3.71$ to 6.02$)$ & 0.64 & $-1.33(-3.94$ to 1.26$)$ & 0.31 & $1.97(-0.98$ to 4.93$)$ & 0.19 \\
\hline
\end{tabular}


Table 3 (Continued).

\begin{tabular}{|c|c|c|c|c|c|c|c|}
\hline $\begin{array}{l}\text { Echocardiographic } \\
\text { Parameters }\end{array}$ & $\begin{array}{l}\text { MHNW } \\
(n=345)\end{array}$ & MHO $(n=60)$ & p-value & MUNW (n=638) & p-value & MUO (n=369) & p-value \\
\hline \multicolumn{8}{|l|}{ A peak, $\mathrm{cm} / \mathrm{s}$} \\
\hline Model I & Reference & $1.32(-4.01$ to 6.66$)$ & 0.62 & $1.87(-0.79$ to 4.54$)$ & 0.16 & 7.71 (4.76 to I0.65) & $<0.0001$ \\
\hline Model 2 & Reference & $1.42(-3.9 \mid$ to 6.76$)$ & 0.60 & $1.62(-1.26$ to $4.5 \mathrm{I})$ & 0.26 & 6.85 (3.60 to 10.10$)$ & $<0.0001$ \\
\hline \multicolumn{8}{|l|}{$e^{\prime}$ peak, $\mathrm{cm} / \mathrm{s}$} \\
\hline Model I & Reference & $-0.25(-0.73$ to 0.22$)$ & 0.29 & $\begin{array}{l}-0.48(-0.71 \text { to } \\
-0.25)\end{array}$ & $<0.0001$ & $\begin{array}{l}-0.63(-0.89 \text { to } \\
-0.37)\end{array}$ & $<0.0001$ \\
\hline Model 2 & Reference & $-0.20(-0.68$ to 0.27$)$ & 0.39 & $\begin{array}{l}-0.39(-0.64 \text { to } \\
-0.14)\end{array}$ & 0.001 & $\begin{array}{l}-0.50(-0.79 \text { to } \\
-0.21)\end{array}$ & 0.0006 \\
\hline \multicolumn{8}{|l|}{$a^{\prime}$ peak, $\mathrm{cm} / \mathrm{s}$} \\
\hline Model I & Reference & $0.11(-0.50$ to 0.73$)$ & 0.71 & $-0.03(-0.35$ to 0.27$)$ & 0.80 & $-0.24(-0.58$ to 0.09$)$ & 0.16 \\
\hline Model 2 & Reference & $0.19(-0.4 \mathrm{I}$ to $0.8 \mathrm{I})$ & 0.52 & $0.21(-0.12$ to 0.55$)$ & 0.21 & $0.06(-0.30$ to 0.44$)$ & 0.72 \\
\hline
\end{tabular}

Notes: Results presented as effect estimate $\pm 95 \% \mathrm{Cl}$, effect estimate considered significant if $\alpha \leq 0.003$. Model I is adjusted for age and sex. Model 2 is adjusted for Model I and sport activity, current smoking and use of medication for hypertension, diabetes or hyperlipidemia.

Abbreviations: LA, left atrium; LV, left ventricle; LVMI, LV mass index; IVSd, interventricular septal diameter; LVPWd, LV posterior wall diameter; LVVd/LVVs, LV enddiastolic/end-systolic volume; LVDd/LVDs, LV end-diastolic/end-systolic diameter; EF, ejection fraction; Cl, confidence interval.

1.53-2.67)), and sTNF-RI (2.22 (95\% CI: 1.62-3.05)) compared to the other phenotypes. For the MUNW group, a more attenuated effect was found, hsCRP (1.33 (95\% CI: 1.14-1.55)), IL-6 (1.26 (95\% CI: 1.08-1.48)) and sTNF-RI (1.36 (95\% CI: 1.13-1.64)), while the MUO group had similar odds ratios, with overlapping confidence intervals, as the MHO group.

\section{Discussion}

In our study of elderly individuals, we explored the association between cardiac geometry, function and inflammation in 4 subgroups based on presence of obesity and metabolic abnormalities. The MHO phenotype showed associations with LA diameter and volume, along with LVMI, parameters usually associated with cardiac remodeling. We additionally identified, after age and sex adjustment, that there were higher odds ratios for inflammatory biomarkers for the MHO phenotype compared to the MHNW phenotype. We postulate that chronic, low-grade inflammation in the elderly, obese, but metabolically healthy individuals is one of the factors that propagates cardiac remodeling, and has its origin in the visceral adipose tissue.

\section{Echocardiographic Characteristics of the 4 Phenotypes}

Echocardiography detects myocardial geometric alterations before the onset of hemodynamic abnormalities. Individuals with obesity and/or metabolic syndrome are known to have changes in cardiac structure and function, such as LV hypertrophy or diastolic dysfunction. LVMI has been shown to predict future risk of cardiovascular events in both men and women. ${ }^{26}$ These subclinical changes precede the development of heart failure, especially the development of heart failure with preserved ejection fraction. ${ }^{27}$ For the MHO group, the adjusted regression models showed an association with LA size and volume, as well as LVMI, but not with parameters for left ventricular diastolic (e' or E/e' ratio) or systolic function (EF). Reports from previous studies are conflicting. A study by Wang et al found that MHO individuals had both systolic and diastolic dysfunction. ${ }^{8}$ Additionally, a study by Park et al reported associations with LVMI and LV diastolic function in MHO individuals, but not with LV systolic function. ${ }^{9}$ On the other hand, the study by Dobson et al, reported that MHO individuals had normal myocardial performance and concluded that the negative influence on cardiac structure and function originates only from the metabolic abnormalities. ${ }^{10}$ However, with a very limited sample size $(n=15)$, the lack of evidence could be due to the study being under-powered to detect any real difference. The limited number of available studies and differences in the definition of the MHO phenotype makes reaching a consensus difficult.

\section{Visceral Adipose Tissue as a Site of Subclinical Chronic Inflammation}

It has been suggested that MHO individuals have a different body fat distribution that accounts for their 


\section{a. Model 1 - hsCRP}

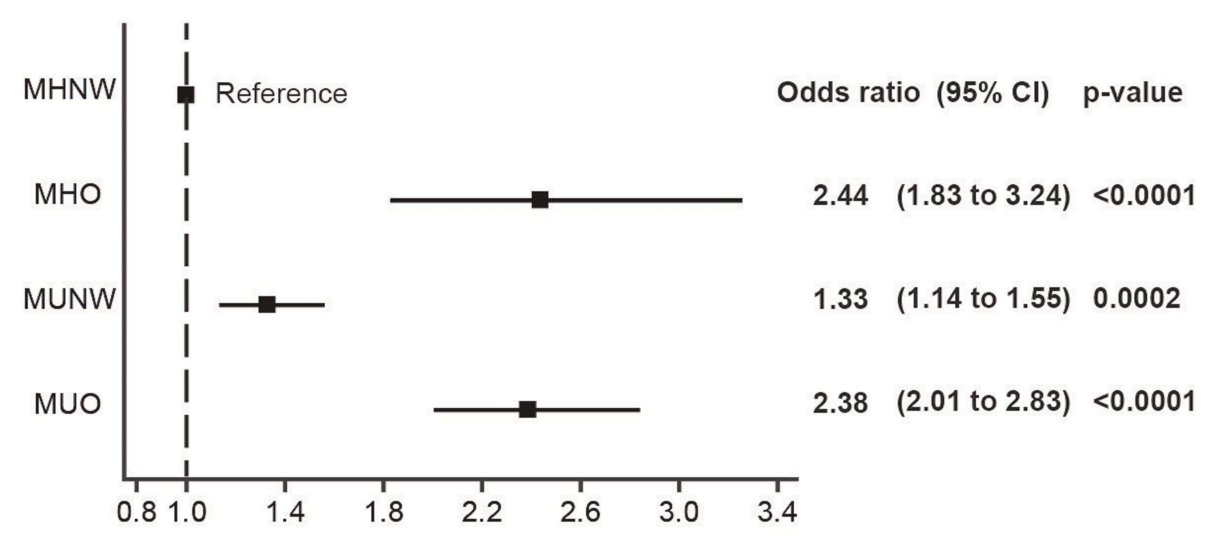

c. Model 1 - IL-6

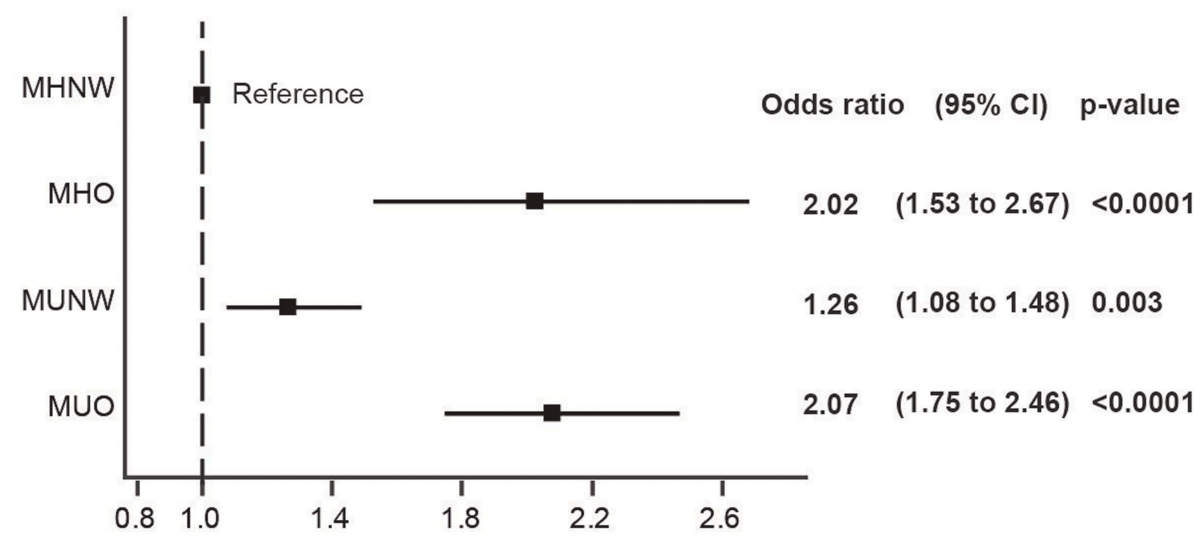

\section{e. Model 1 - sTNF-RI}

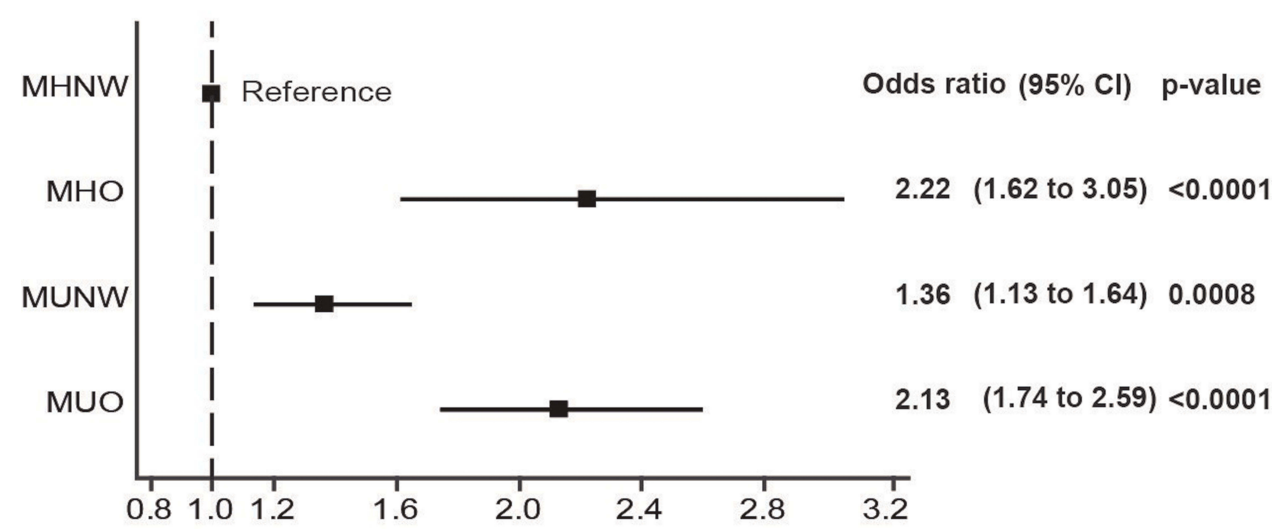

Figure 2 Forest plots of odds ratios and $95 \% \mathrm{Cl}$ for inflammatory biomarkers. Model I - age and sex adjusted. Odds ratios shown are for a I-SD increment. Abbreviations: MHNW, metabolically healthy, normal weight; MHO, metabolically healthy obese; MUNW, metabolically unhealthy, normal weight; MUO, metabolically unhealthy obese; $\mathrm{Cl}$, confidence interval. 
favorable metabolic profile. ${ }^{28}$ Visceral adipose tissue is recognized as a risk factor for diabetes and cardiovascular disease (CVD), whereas subcutaneous adipose tissue is considered as more benign. ${ }^{15,29,30}$ Genome-wide association studies have shown that impaired capacity to store fat in the subcutaneous adipose tissue can lead to metabolically harmful ectopic fat storage. Several adiposityincreasing variants that have protective effects on cardiometabolic outcomes, such as IRS1, COBLL1/ GRB14, PLA2G6 and TOMM40, have been identified. ${ }^{31}$

Waist circumference is an alternative, clinically important indicator for visceral adiposity and a possible site for subclinical systemic inflammation. ${ }^{11}$ In our cohort, MHO individuals presented with a higher waist circumference compared to the MHNW group. Visceral obesity has been linked with inflammatory biomarkers IL-6 and hsCRP, ${ }^{32}$ as well as, increased oxidative stress. ${ }^{12}$ This dysfunctional tissue, constituted by adipocytes and cytokine-producing macrophages, is hypothesized to contribute to the development of metabolic complications. Increase in the number of macrophages is characteristic for obese individuals and is responsible for TNF- $\alpha$ and IL-6 expression. ${ }^{29}$ sTNF-RI is an inhibitory biomarker for TNF- $\alpha$ and increases as a response to more TNF- $\alpha$ production. ${ }^{33}$ A state of sustained metabolic health in obese individuals was shown only when they maintained a lower waist circumference, as reported by a previous cohort study. ${ }^{34}$ The factors that induce immune cells to infiltrate adipose tissue are unknown, but may be related to free fatty acids released from adipocytes that activate macrophages, which in turn increase cytokine levels, such as TNF- $\alpha$, that perpetuates and sustains lipolysis and fatty acid release. ${ }^{35,36}$

\section{Potential Mechanisms of Action}

Several studies have shown that MHO individuals have a different inflammation profile in comparison to MHNW individuals. The study by Iglesias Molli et al concluded that MHO is a pro-inflammatory state. ${ }^{15}$ Chronic inflammation is a possible explanation for the association with echocardiography parameters for LV hypertrophy and diastolic dysfunction. ${ }^{37,38}$ Inflammatory biomarkers act in a negative inotropic manner and cause changes in turnover of the extracellular matrix resulting in myocardial fibrosis. Elevated concentrations of TNF- $\alpha$, as well as IL-6, have cardio-depressive properties and cause apoptosis. ${ }^{33}$ The development of progressive cardiomyocyte apoptosis plays a critical role on LV geometry and adverse cardiac remodeling that occurs in the setting of sustained inflammation. ${ }^{39,40}$ In addition, obesity is associated with increased gut permeability to bacteria and their products, which further propagates systemic inflammation. ${ }^{41}$ Opposite this, one study reported that MHO individuals present with a favorable inflammation profile. However, this study had a different definition for the MHO phenotype, with younger participants, without reporting waist circumference measurements. ${ }^{42}$ Obesity-related or unrelated inflammation is managed differently in young versus elderly individuals. ${ }^{16}$ Our results have shown similar odds for inflammatory biomarkers between MHO and MUO elderly individuals, however more attenuated odds for the MUNW phenotype, compared with MHNW. The implication of this finding is that increased inflammation stems from the adipose tissue, and is less influenced by other metabolical abnormalities.

Several studies have explored the longitudinal aspect of the MHO phenotype and have concluded that the MHO state may be transient and over time MHO individuals will develop metabolic abnormalities and clinical disease ${ }^{43,44}$ however, it can still be an opportunity for primary prevention for CVDs. ${ }^{45}$ An important finding is that the role of chronic inflammation was ascertained longitudinally as an important predictor for CVD risk in the MHO group. ${ }^{46}$ Finally, physical fitness is an important effect-modifier of CVD risk. ${ }^{47}$ In our cohort, MHO individuals had the highest percentage $(\approx 57 \%)$ of reported current sport activity. Several studies have shown that MHO individuals have higher cardiorespiratory fitness ${ }^{48,49}$ and physical activity ${ }^{50}$ that act as protective factors. Obesity imposes a persistent lipid burden on muscle mitochondria, and the subsequent acylcarnitine accumulation and mitochondrial dysfunction can be improved with physical exercise. ${ }^{51}$

\section{Strengths and Limitations}

Our population-based cohort, representative of the elderly population, is well phenotyped and contains a comprehensive set of cardiac structural and functional parameters that offer robustness to our results. However, they have to be interpreted by taking certain limitations into account. The study participants were not required to fast before blood sample collection, which could influence the levels of glucose and incur misclassification bias. However, $95 \%$ of participants reported abstaining from food and beverages consumption more than 2 hours before coming to the study center, so we expect this bias to be small. Due to the relatively small sample size of the MHO group, separate analysis for gender differences was not performed. The 
potential implications are important since research has shown that the association between visceral adipose tissue and inflammatory biomarkers, such as TNF- $\alpha$ and IL-6, is weaker in older men. ${ }^{52}$ In addition, waist circumference is only an indirect measurement of visceral adipose tissue. This tissue is not homogeneous and is best analyzed by CT, ultrasound or whole body DXA scans. Other parameters for assessing systolic function, including longitudinal strain, strain rate, and LV dyssynchrony assessed by speckle tracking, were not measured. Future studies should implement the measurement of these parameters, since abdominal obesity was shown to be associated with systolic mechanics. ${ }^{8}$ Finally, considering the cross-sectional nature of the study, causal inferences cannot be determined.

\section{Conclusion}

To summarize, the MHO phenotype was characterized with greater LA diameter and volume, greater LVMI, and higher levels of inflammatory biomarker concentrations compared to the MHNW group. The lack of metabolic burden offers advantages that are inhibitory in the progression of cardiac remodeling in an aging population. Further examination of pathophysiological mechanisms could unravel new points of care and treatment.

\section{Acknowledgments}

We are thankful to all participants, study nurses, clerical staff and medical professionals who made the CARLA study possible. The authors also wish to thank Jens Höpner and Sandra Steensels for helpful discussions during the preparation of the manuscript. We acknowledge the financial support of the Open Access Publication Fund of the Martin-Luther-University Halle-Wittenberg.

\section{Funding}

The CARLA study was supported by a grant from the German Research Foundation (DFG) as part of the Collaborative Research Project 598 "Heart failure in the elderly-cellular mechanisms and therapy" at the Medical Faculty of the Martin-Luther-University Halle-Wittenberg. Additional funding was obtained from the Wilhelm-Roux Programme of the Martin-Luther-University Halle-Wittenberg (FKZ 14/41, 16/ 19), the Ministry of Education and Cultural Affairs of SaxonyAnhalt (MKCARLA-MLU-2011), and the German Federal Employment Office.

\section{Disclosure}

The Authors declare no conflicts of interest in this work.

\section{References}

1. Primeau V, Coderre L, Karelis AD, et al. Characterizing the profile of obese patients who are metabolically healthy. Int J Obes. 2011;35 (7):971-981. doi:10.1038/ijo.2010.216

2. Karelis AD, Brochu M, Rabasa-Lhoret R. Can we identify metabolically healthy but obese individuals (MHO)? Diabetes Metab. 2004;30(6):569-572. doi:10.1016/S1262-3636(07)70156-8

3. Iacobellis G, Ribaudo MC, Zappaterreno A, Iannucci CV, Leonetti F. Prevalence of uncomplicated obesity in an Italian obese population. Obes Res. 2005;13(6):1116-1122. doi:10.1038/oby.2005.130

4. Korduner J, Bachus E, Jujic A, Magnusson M, Nilsson PM. Metabolically healthy obesity (MHO) in the Malmo diet cancer study - epidemiology and prospective risks. Obes Res Clin Pract. 2019;13(6):548-554. doi:10.1016/j.orcp.2019.10.002

5. Al-Khalidi B, Kimball SM, Kuk JL, Ardern CI. Metabolically healthy obesity, vitamin $\mathrm{D}$, and all-cause and cardiometabolic mortality risk in NHANES III. Clin Nutr. 2019;38(2):820-828. doi:10.1016/j.clnu.2018.02.025

6. Sims EA. Are there persons who are obese, but metabolically healthy? Metabolism. 2001;50(12):1499-1504. doi:10.1053/meta.2001.27213

7. Bluher M. The distinction of metabolically 'healthy' from 'unhealthy' obese individuals. Curr Opin Lipidol. 2010;21 (1):38-43. doi:10.1097/MOL.0b013e3283346ccc

8. Wang YC, Liang CS, Gopal DM, et al. Preclinical systolic and diastolic dysfunctions in metabolically healthy and unhealthy obese individuals. Circ Heart Fail. 2015;8(5):897-904. doi:10.1161/ CIRCHEARTFAILURE.114.002026

9. Park J, Kim SH, Cho GY, et al. Obesity phenotype and cardiovascular changes. J Hypertens. 2011;29(9):1765-1772. doi:10.1097/HJH.0b 013e32834a50f3

10. Dobson R, Burgess MI, Sprung VS, et al. Metabolically healthy and unhealthy obesity: differential effects on myocardial function according to metabolic syndrome, rather than obesity. Int J Obes. 2016;40 (1):153-161. doi:10.1038/ijo.2015.151

11. Hamer M, Bell JA, Sabia S, Batty GD, Kivimaki M. Stability of metabolically healthy obesity over 8 years: the English longitudinal study of ageing. Eur $J$ Endocrinol. 2015;173(5):703-708. doi:10.1530/EJE-15-0449

12. Pou KM, Massaro JM, Hoffmann U, et al. Visceral and subcutaneous adipose tissue volumes are cross-sectionally related to markers of inflammation and oxidative stress: the Framingham heart study. Circulation. 2007;116(11):1234-1241. doi:10.1161/CIRCULATIONAHA.107.710 509

13. Ortega FJ, Fernandez-Real JM. Inflammation in adipose tissue and fatty acid anabolism: when enough is enough! Hormon Metab Res. 2013;45(13):1009-1019. doi:10.1055/s-0033-1358690

14. Chait A, den Hartigh LJ. Adipose tissue distribution, inflammation and its metabolic consequences, including diabetes and cardiovascular disease. Front Cardiovasc Med. 2020;7:22.

15. Iglesias Molli AE, Penas Steinhardt A, Lopez AP, et al. Metabolically healthy obese individuals present similar chronic inflammation level but less insulin-resistance than obese individuals with metabolic syndrome. PLoS One. 2017;12(12):e0190528. doi:10.1371/journal.pone.0190528

16. Alam I, Ng TP, Larbi A. Does inflammation determine whether obesity is metabolically healthy or unhealthy? The aging perspective. Mediators Inflamm. 2012;2012:456456. doi:10.1155/2012/456456

17. Greiser KH, Kluttig A, Schumann B, et al. Cardiovascular disease, risk factors and heart rate variability in the elderly general population: design and objectives of the CARdiovascular disease, living and ageing in Halle (CARLA) study. BMC Cardiovasc Disord. 2005;5:33. doi:10.1186/1471-2261-5-33

18. Greiser KH, Kluttig A, Schumann B, et al. Cardiovascular diseases, risk factors and short-term heart rate variability in an elderly general population: the CARLA study 2002-2006. Eur J Epidemiol. 2009;24 (3):123-142. doi:10.1007/s10654-009-9317-z 
19. Levey AS, Stevens LA, Schmid $\mathrm{CH}$, et al. A new equation to estimate glomerular filtration rate. Ann Intern Med. 2009;150 (9):604-612. doi:10.7326/0003-4819-150-9-200905050-00006

20. Gottdiener JS, Bednarz J, Devereux R, et al. American Society of Echocardiography recommendations for use of echocardiography in clinical trials. J Am Soc Echocardiogr. 2004;17(10):1086-1119. doi:10.1016/j.echo.2004.07.013

21. Lang RM, Bierig M, Devereux RB, et al. Recommendations for chamber quantification: a report from the American Society of Echocardiography's Guidelines and Standards Committee and the Chamber Quantification Writing Group, developed in conjunction with the European Association of Echocardiography, a branch of the European Society of Cardiology. $\mathrm{J} \mathrm{Am} \mathrm{Soc} \mathrm{Echocardiogr.}$ 2005;18(12):1440-1463. doi:10.1016/j.echo.2005.10.005

22. Chirinos JA, Segers P, De Buyzere ML, et al. Left ventricular mass: allometric scaling, normative values, effect of obesity, and prognostic performance. Hypertension. 2010;56(1):91-98. doi:10.1161/ HYPERTENSIONAHA. 110.150250

23. Alberti KG, Eckel RH, Grundy SM, et al. Harmonizing the metabolic syndrome: a joint interim statement of the International Diabetes Federation Task Force on Epidemiology and Prevention; National Heart, Lung, and Blood Institute; American Heart Association; World Heart Federation; International Atherosclerosis Society; and International Association for the Study of Obesity. Circulation. 2009;120 (16):1640-1645. doi:10.1161/CIRCULATIONAHA.109.192644

24. Demirtas H, Freels SA, Yucel RM. Plausibility of multivariate normality assumption when multiply imputing non-Gaussian continuous outcomes: a simulation assessment. J Stat Comput Simul. 2008;78 (1):69-84. doi:10.1080/10629360600903866

25. Bodner TE. What improves with increased missing data imputations? Struct Equ Modeling. 2008;15(4):651-675. doi:10.1080/107055108 02339072

26. Liao Y, Cooper RS, Mensah GA, McGee DL. Left ventricular hypertrophy has a greater impact on survival in women than in men. Circulation. 1995;92(4):805-810. doi:10.1161/01.CIR.92.4.805

27. Morkedal B, Vatten LJ, Romundstad PR, Laugsand LE, Janszky I. Risk of myocardial infarction and heart failure among metabolically healthy but obese individuals: HUNT (Nord-Trondelag Health Study), Norway. J Am Coll Cardiol. 2014;63(11):1071-1078. doi:10. 1016/j.jacc.2013.11.035

28. Mathew H, Farr OM, Mantzoros CS. Metabolic health and weight: understanding metabolically unhealthy normal weight or metabolically healthy obese patients. Metabolism. 2016;65(1):73-80. doi:10.1016/j.metabol.2015.10.019

29. Weisberg SP, McCann D, Desai M, Rosenbaum M, Leibel RL, Ferrante AW. Obesity is associated with macrophage accumulation in adipose tissue. J Clin Invest. 2003;112(12):1796-1808. doi:10. 1172/JCI200319246

30. Altintas MM, Azad A, Nayer B, et al. Mast cells, macrophages, and crown-like structures distinguish subcutaneous from visceral fat in mice. J Lipid Res. 2011;52(3):480-488. doi:10.1194/jlr.M011338

31. Loos RJF, Kilpelainen TO. Genes that make you fat, but keep you healthy. J Intern Med. 2018;284(5):450-463. doi:10.1111/joim.12827

32. Park HS, Park JY, Yu R. Relationship of obesity and visceral adiposity with serum concentrations of CRP, TNF-alpha and IL-6. Diabetes Res Clin Pract. 2005;69(1):29-35. doi:10.1016/j.diabres. 2004.11.007

33. Aderka D. The potential biological and clinical significance of the soluble tumor necrosis factor receptors. Cytokine Growth Factor Rev. 1996;7(3):231-240. doi:10.1016/S1359-6101(96)00026-3

34. Appleton SL, Seaborn CJ, Visvanathan R, et al. Diabetes and cardiovascular disease outcomes in the metabolically healthy obese phenotype: a cohort study. Diabetes Care. 2013;36(8):2388-2394. doi: $10.2337 / \mathrm{dc} 12-1971$
35. Schaffler A, Scholmerich J. Innate immunity and adipose tissue biology. Trends Immunol. 2010;31(6):228-235. doi:10.1016/j.it.2010. 03.001

36. Shi H, Kokoeva MV, Inouye K, Tzameli I, Yin H, Flier JS. TLR4 links innate immunity and fatty acid-induced insulin resistance. J Clin Invest. 2006;116(11):3015-3025. doi:10.1172/JCI28898

37. Masiha S, Sundstrom J, Lind L. Inflammatory markers are associated with left ventricular hypertrophy and diastolic dysfunction in a population-based sample of elderly men and women. J Hum Hypertens. 2013;27(1):13-17. doi:10.1038/jhh.2011.113

38. Dinh W, Futh R, Nick1 W, et al. Elevated plasma levels of TNF-alpha and interleukin-6 in patients with diastolic dysfunction and glucose metabolism disorders. Cardiovasc Diabetol. 2009;8:58. doi:10.1186/ 1475-2840-8-58

39. Bozkurt B, Kribbs SB, Clubb FJ, et al. Pathophysiologically relevant concentrations of tumor necrosis factor-alpha promote progressive left ventricular dysfunction and remodeling in rats. Circulation. 1998;97(14):1382-1391. doi:10.1161/01.CIR.97.14.1382

40. Hamid T, Xu Y, Ismahil MA, et al. TNF receptor signaling inhibits cardiomyogenic differentiation of cardiac stem cells and promotes a neuroadrenergic-like fate. Am J Physiol Heart Circ Physiol. 2016;311 (5):H1189-H1201. doi:10.1152/ajpheart.00904.2015

41. Brun P, Castagliuolo I, Di Leo V, et al. Increased intestinal permeability in obese mice: new evidence in the pathogenesis of nonalcoholic steatohepatitis. Am $J$ Physiol Gastrointest Liver Physiol. 2007;292(2):G518-525. doi:10.1152/ajpgi.00024.2006

42. Shin MJ, Hyun YJ, Kim OY, Kim JY, Jang Y, Lee JH. Weight loss effect on inflammation and LDL oxidation in metabolically healthy but obese (MHO) individuals: low inflammation and LDL oxidation in MHO women. Int J Obes. 2006;30(10):1529-1534. doi:10.1038/sj. ijo.0803304

43. Echouffo-Tcheugui JB, Short MI, Xanthakis V, et al. Natural history of obesity subphenotypes: dynamic changes over two decades and prognosis in the Framingham heart study. J Clin Endocrinol Metab. 2019;104(3):738-752. doi:10.1210/jc.2018-01321

44. Espinosa De Ycaza AE, Donegan D, Jensen MD. Long-term metabolic risk for the metabolically healthy overweight/obese phenotype. Int J Obes. 2018;42(3):302-309. doi:10.1038/ijo.2017.233

45. Mongraw-Chaffin M, Foster MC, Anderson CAM, et al. Metabolically healthy obesity, transition to metabolic syndrome, and cardiovascular risk. J Am Coll Cardiol. 2018;71(17):1857-1865. doi:10.1016/j. jacc.2018.02.055

46. Kouvari M, Panagiotakos DB, Yannakoulia M, et al. Transition from metabolically benign to metabolically unhealthy obesity and 10-year cardiovascular disease incidence: the ATTICA cohort study. Metabolism. 2019;93:18-24. doi:10.1016/j.metabol.2019. 01.003

47. Hamer M, Ingle L, Carroll S, Stamatakis E. Physical activity and cardiovascular mortality risk: possible protective mechanisms? Med Sci Sports Exerc. 2012;44(1):84-88. doi:10.1249/MSS.0b013e 3182251077

48. Poelkens F, Eijsvogels TM, Brussee P, Verheggen RJ, Tack CJ, Hopman MT. Physical fitness can partly explain the metabolically healthy obese phenotype in women. Exp Clin Endocrinol Diabetes. 2014;122(2):87-91. doi:10.1055/s-0033-1363686

49. Ingle L, Swainson M, Brodie D, Sandercock GR. Characterization of the metabolically healthy phenotype in overweight and obese British men. Prev Med. 2017;94:7-11. doi:10.1016/j.ypmed. 2016.11.001

50. Roberson LL, Aneni EC, Maziak W, et al. Beyond BMI: the "Metabolically healthy obese" phenotype \& its association with clinical/subclinical cardiovascular disease and all-cause mortality a systematic review. BMC Public Health. 2014;14:14. doi:10.1186/ $1471-2458-14-14$ 
51. Koves TR, Li P, An J, et al. Peroxisome proliferator-activated receptor-gamma co-activator 1alpha-mediated metabolic remodeling of skeletal myocytes mimics exercise training and reverses lipid-induced mitochondrial inefficiency. J Biol Chem. 2005;280 (39):33588-33598. doi:10.1074/jbc.M507621200
52. Schousboe JT, Langsetmo L, Schwartz AV, et al. Comparison of associations of DXA and CT visceral adipose tissue measures with insulin resistance, lipid levels, and inflammatory markers. $J$ Clin Densitometr. 2017;20(2):256-264. doi:10.1016/j.jocd.2017.01.004

Diabetes, Metabolic Syndrome and Obesity: Targets and Therapy

\section{Publish your work in this journal}

Diabetes, Metabolic Syndrome and Obesity: Targets and Therapy is an international, peer-reviewed open-access journal committed to the rapid publication of the latest laboratory and clinical findings in the fields of diabetes, metabolic syndrome and obesity research. Original research, review, case reports, hypothesis formation, expert opinion and commentaries are all considered for publication. The manuscript management system is completely online and includes a very quick and fair peer-review system, which is all easy to use. Visit http://www.dovepress.com/testimonials.php to read real quotes from published authors. 